\title{
USING COUPLED NONNEGATIVE MATRIX FACTORIZATION (CNMF) UN-MIXING FOR HIGH SPECTRAL AND SPATIAL RESOLUTION DATA FUSION TO ESTIMATE URBAN IMPERVIOUS SURFACE AND URBAN ECOLOGICAL ENVIRONMENT
}

\author{
T. Wang ${ }^{\mathrm{a}, \mathrm{b}, *}, \mathrm{H}$. Zhang ${ }^{\mathrm{a}, \mathrm{b}}, \mathrm{H}$. Lin ${ }^{\mathrm{a}, \mathrm{b}}$ \\ ${ }^{a}$ Institute of Space and Earth Information Science, The Chinese University of Hong Kong, New Territories, Hong Kong \\ - (tingwang_ISEIS, stevenzhang, huilin)@cuhk.edu.hk \\ ${ }^{\mathrm{b}}$ Shenzhen Research Institute, The Chinese University of Hong Kong, Shenzhen, 518057, China
}

Commission III, WG III/6

KEY WORDS: Data fusion; Impervious surface; Coupled Nonnegative Matrix Factorization

\begin{abstract}
:
Remote sensing techniques have great potential in providing accurate and timely information in urban areas. Estimation of impervious surfaces has increasingly roused widely interests of researchers in monitoring urban development and determining the overall environmental health of a watershed. However, studies on the impervious surface using multi-spectral imageries is insufficient and inaccurate due to the complexity of urban infrastructures base on the need to further recognize these impervious surface materials in a finer scale. Hyperspectral imageries have been proved to be sensitive to subtle spectral differences thus capable to exquisitely discriminate these similar materials while limited to the low spatial resolution. Coupled nonnegative matrix factorization (CNMF) unmixing method is one of the most physically straightforward and easily complemented hyperspectral pan-sharpening methods that could produce fused data with both high spectral and spatial resolution. This paper aimed to exploit the latent capacity and tentative validation of CNMF on the killer application of mapping urban impervious surfaces in complexed metropolitan environments like Hong Kong. Experiments showed that the fusion of high spectral and spatial resolution image could provide more accurate and comprehensive information on urban impervious surface estimation.
\end{abstract}

\section{INTRODUCTION}

Remote sensing techniques have great potential in providing accurate and timely information in urban areas and a great number of studies have focused on remote sensing of urban environments and their specific land cover types [1, 2]. A prominent change associated with urbanization is the expansion of impervious surface (IS) and the knowledge of IS, especially the magnitude, location, geometry, spatial pattern of impervious surfaces in urban area, is significant to a range of issues and themes in environmental science central to global environmental change and human-environment interactions [3].

Impervious surfaces can be generally defined as any material of natural or anthropogenic source - that prevents the infiltration of water into the soil and thereby changing the flow dynamics, sedimentation load and pollution profile of storm water runoff [4]. It is significant for urban planning and environmental and resources management and remote sensing has provided an efficient, affordable, and timely manner to detect and monitor IS [5]. It is certified that IS have a great impact on the urban solar energy balance, air quality, nonpoint source water pollution, storm runoff processes, and in addition to environment study, IS have also been identified as a key factor in many socioeconomic studies, such as the measurement of urban growth, the estimation of population distribution, and variation of housing prices [6]. However, due to the complexity of urban landscapes and inherent and stochastic human activities, coarse resolution urban maps are difficult to use for many applications from local to a regional study. Medium resolution satellite imagery possesses unique advantages more accurately especially for that sensor on board the Landsat series of satellites have been providing earth observation data continuously since the early 1970s [7]. Studies from Landsat imageries have been applied in numerous urbanization process different scale [8] while IS estimation using multi-spectral imageries is insufficient and inaccurate due to the complexity of urban infrastructures base on the need to further recognize these impervious surface materials in a finer scale $[9$, $10]$.

There are various kinds of reasons that making urban environments exhibit a higher dimensionality than multi-spectral images could provide with [11]. Bearing brunt of the problem is the presence of spectrally ambiguous materials. Hyperspectral imageries have been proved to be sensitive to subtle spectral differences thus capable to exquisitely discriminate these similar materials while limited to the low spatial resolution [12]. Due to the inevitable trade-off between spatial resolution, spectral resolution, and signal-to-noise ratio (SNR), space-borne imaging spectrometers are usually designed to provide data with a moderate ground sampling distance (GSD) like Hyperion [13]. Clearly, this spatial resolution of the space-borne hyperspectral sensor cannot satisfy the demand of exquisite estimation of urban IS. Data fusion can be performed to generate high spatial resolution hyperspectral data to allow for various new applications potentially conducted on a high-spatial-resolution airborne imaging system only, which is usually very expensive $[14,15]$. As have been reviewed, a variety of data fusion techniques - pan sharpening have been developed in the past two decades; representative techniques can be roughly categorized as follows: 1) component substitution (CS); 2) multiresolution analysis (MRA); 3) un-mixing; and 4) Bayesian-based approaches. Among these proposed HS-MS methods, Coupled nonnegative matrix factorization (CNMF) un-mixing method is

\footnotetext{
* Corresponding author: Ting Wang, tingwang_ISEIS@cuhk.edu.hk
} 
one of the most physically straightforward and easily complemented hyperspectral pan-sharpening methods that could effectively and efficiently produce fused data as it estimates the endmember and abundance matrices via alternating spectral unmixing based on NMF under the constraints of an observation model which incorporates both the spectral response function (SRF) and point spread function (PSF) [16].

Resorting to this benchmark fusion method of HS and MS, synthetic urban remotely sensed image equipped with both geometric and spectral information of certain kind of IS materials could be obtained, thus facilitating the applications of exquisite mapping and estimating the urban IS. The remainder of the paper is organized as follows: Section 2 briefly introduces the fusion method; Section 3 illustrates the study area and experiment settings; and finally, a discussion of the results is provided about the objective evaluation of the method on the urban applications.

\section{METHOD}

Before conducting the data fusion, pre-processing of HSI and MSI were accomplished to a routine as following steps:

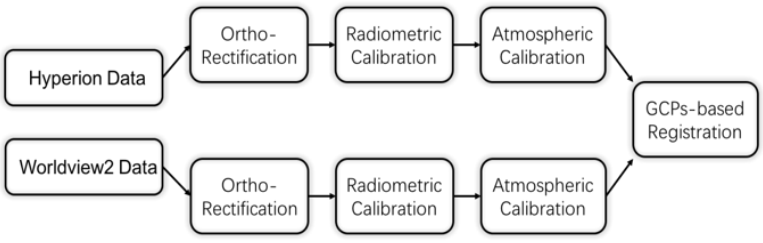

Fig. 1. Pre-processing of HSI and MSI data.

Ortho-rectification is especially significant for the image registration as two different sensors have varied platform altitude and image acquiring time under mismatched sunshine condition status. High spatial resolution image like Worldview2 suffers a lot from distortions from the non-ortho-rectification image and could not be well registered with coarse resolution image. After that, digital numbers of the image are radiometric calibrated to radiance, then atmospheric calibrated to surface reflectance for the image registration step. As the spatial resolution difference of this two image is huge, the accurate registration is quite challenging. The matching of tie-points should be carefully selected to lower the RMSE down as much as possible.

Hyperspectral and multispectral data fusion based on un-mixing are achieved by the estimation of the high-spectral-resolution endmember spectra and the high-spatial-resolution abundance maps from the two data.

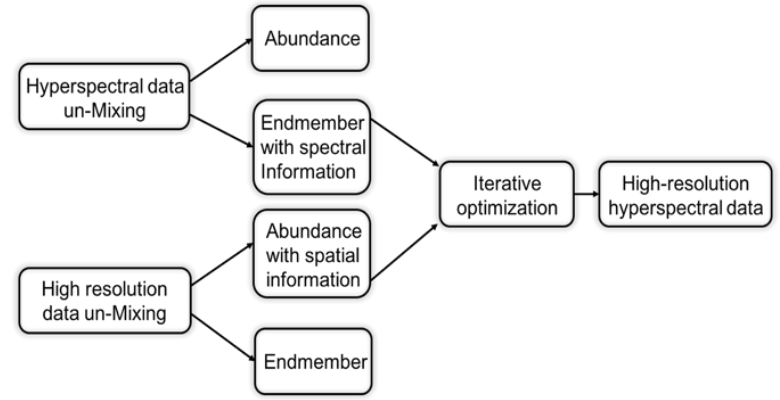

Fig. 2. Fusion working flow of HSI and MSI data.

Non-negative matrix factorization (NMF) [1] has been chosen for un-mixing processing of two datasets as it could effectively factorize a nonnegative hyperspectral data matrix into two nonnegative matrices, one is the identified endmember spectra and another is the corresponding abundances simultaneously without pure pixel assumption $[2,3]$.
Following is the summarized calculate steps of CNMF methods to fuse hyperspectral data and high spatial resolution data:

Algorithm: CNMF un-mixing for hyperspectral and multispectral data fusion

Input: Hyperspectral data $\mathbf{X} \in \mathbb{R}^{\lambda_{h} \times L_{h}}$ and multispectral data $\mathbf{Y} \in \mathbb{R}^{\lambda_{m} \times L_{m}}$.

Output: Two matrices $\mathbf{W} \in \mathbb{R}^{\lambda_{h} \times D}$ and $\mathbf{H} \in \mathbb{R}^{D \times L_{m}}$.

Step1. First NMF of $\mathbf{X}$
1a) Initialize $\mathbf{W}$ by VCA, and update $\mathbf{H}_{h}$ with $\mathbf{W}$ fixed.

1b) Optimize $\mathbf{W}$ and $\mathbf{H}_{h}$.

Step2. NMF of $\mathbf{Y}$

2a) Initialize $\mathbf{W}_{m}$ and update $\mathbf{H}$ with $\mathbf{W}_{m}$ fixed.

2b) Optimize $\mathbf{W}_{m}$ and $\mathbf{H}$.

Step3. Subsequent NMF of $\mathbf{X}$

3a) Initialize $\mathbf{H}_{h}$ and update $\mathbf{W}$ with $\mathbf{H}_{h}$ fixed.

3b) Optimize $\mathbf{W}$ and $\mathbf{H}_{h}$.

Step4. Repeat Steps 2 and 3.

After obtaining image data with both high spatial and spectral resolution, detailed analysis of complicated impervious surface in an urban area could be followed by some benchmark classification and indices method.

\section{EXPERIMENTS}

The study area located in Kowloon peninsula in Hong Kong SAR (Special Administrative Region) of the People's Republic of China. The whole administrative region boundaries are shown in Fig. 4 and the research area is enclosed by a red rectangular. As it is well known that Hong Kong is a fully developed urbanization city full of man-made infrastructure. The urban environment that composed of impervious surfaces and vegetation cover is seriously taken into consideration by local government and citizens. Remote sensing technique has the potential to solve these problems on a large scale and high repetitive rate. It could greatly promote the survey efficiency and save the manpower costs.

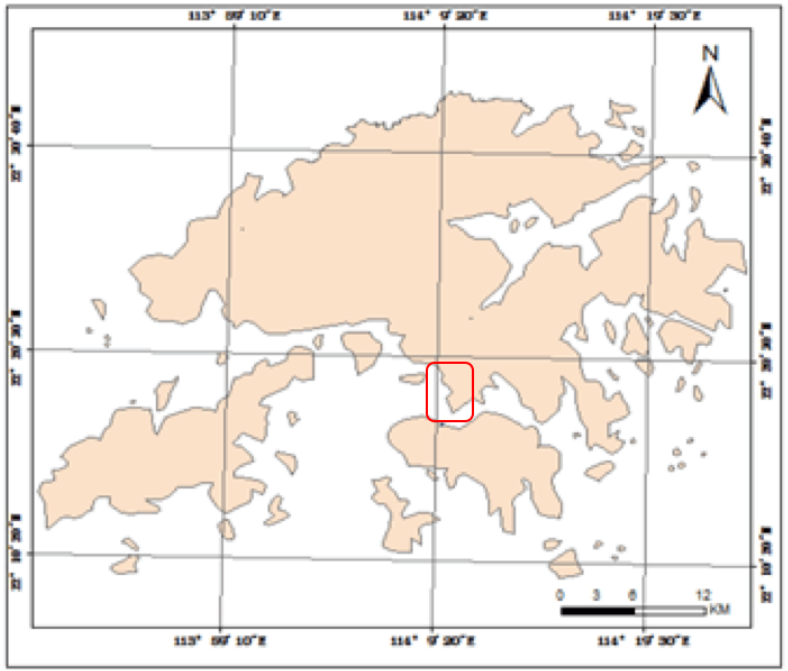

Fig. 3. The administrative scope of Hong Kong and the research area. 
This study area is carefully chosen as the research topic is to test the analysis of urban impervious surface from the fused hyperspectral and high resolution multispectral data. The landscape of the selected region is mostly composed of all kinds of the built-up urban area including a residential area, industrial zone and commercial skyscrapers which could be observed from the false color combination shown in Fig. 4 (b).

The two under fusion remotely sensed imagery comes from Hyperion sensor and Worldview 2 as shown in Fig. 4(a) and Fig. 4(b), respectively. The Hyperion instrument loaded on EO-1 satellite provides a 30 -meter spatial resolution hyperspectral imager capable of resolving 220 spectral bands covering the spectral range from 0.4 to $2.5 \mu \mathrm{m}$. After band selection of discarding water absorption and low signal-to-noise ratio bands, 131 bands covering 0.42 to $1.98 \mu \mathrm{m}$ has been left for data fusion. The Worldview 2 sensor provides a high spatial resolution image resampled to 2-meter spanning 8 multispectral bands, 4 standard colors (red, green, blue, and near-infrared 1) and 4 innovative bands (coastal, yellow, red edge, and near-infrared 2). In this study, only the standard four bands are adopted to test the generalization ability of CNMF, for its potential on other similar sensor platforms. The false color image of registered two datasets is shown in Fig. 4(a) and Fig. 4(b).

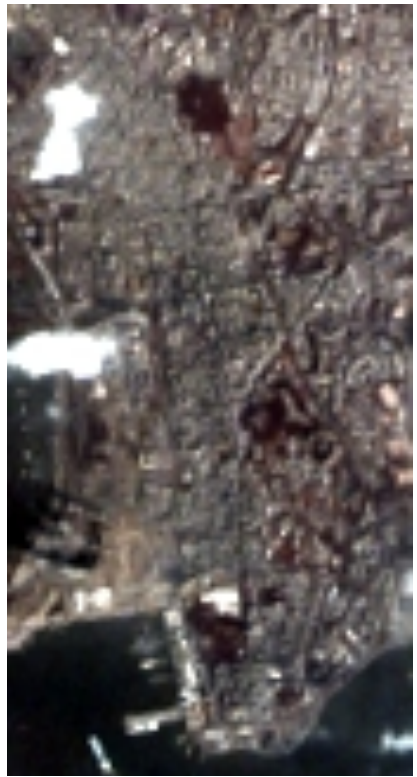

(a) Hyperion hyperspectral image

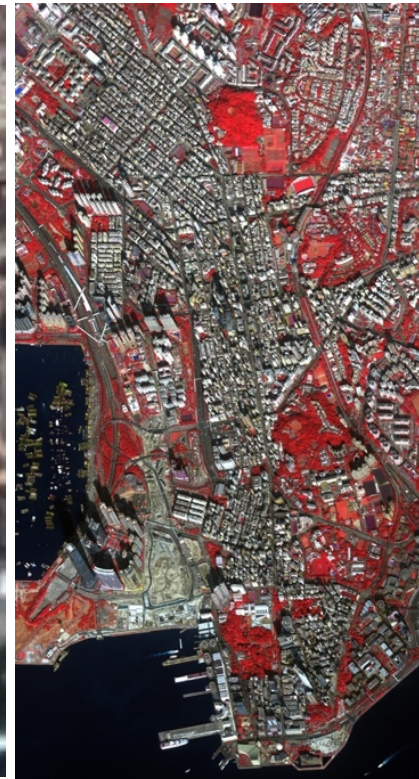

(b) Worldview 2 multispectral high resolution image
Fig. 4. False color combination image of HSI and MSI.

The fusion image with both high spatial and spectral resolution is shown in Fig. 5 with a true color display to reflect its visual performance.

For the evaluation of the fused image, qualitative and quantitative analysis are designed to give out a comprehensive evaluation. Consulting to the benchmark V-I-S model brought in 2003[4], estimating the distribution of impervious surface, a major component of the vegetation - impervious surface - soil model, is important in monitoring urban areas and understanding human activities. We selected a zone out of the study area that composed of complicated surface material, the zoomed hyperspectral cube is shown in Fig.6.

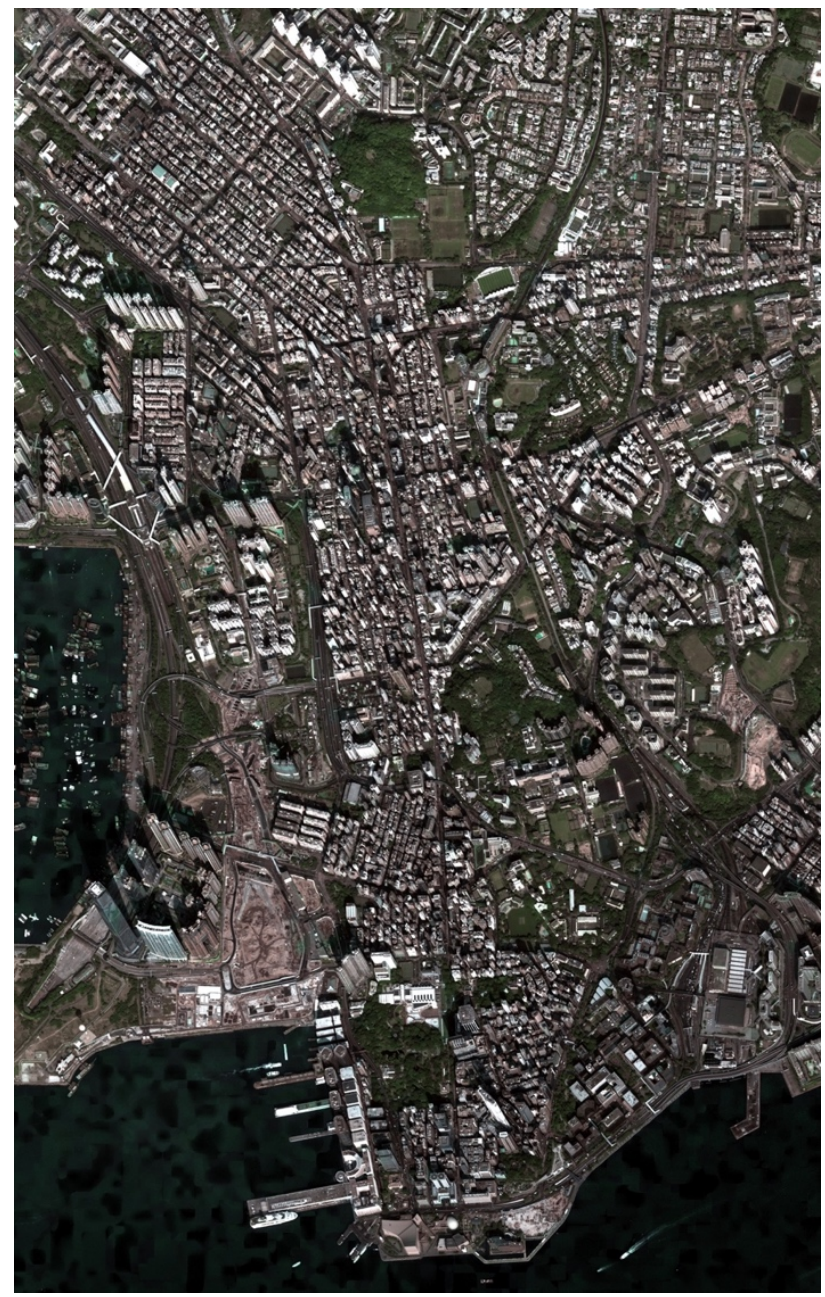

Fig. 5. True color combination image of fused data.

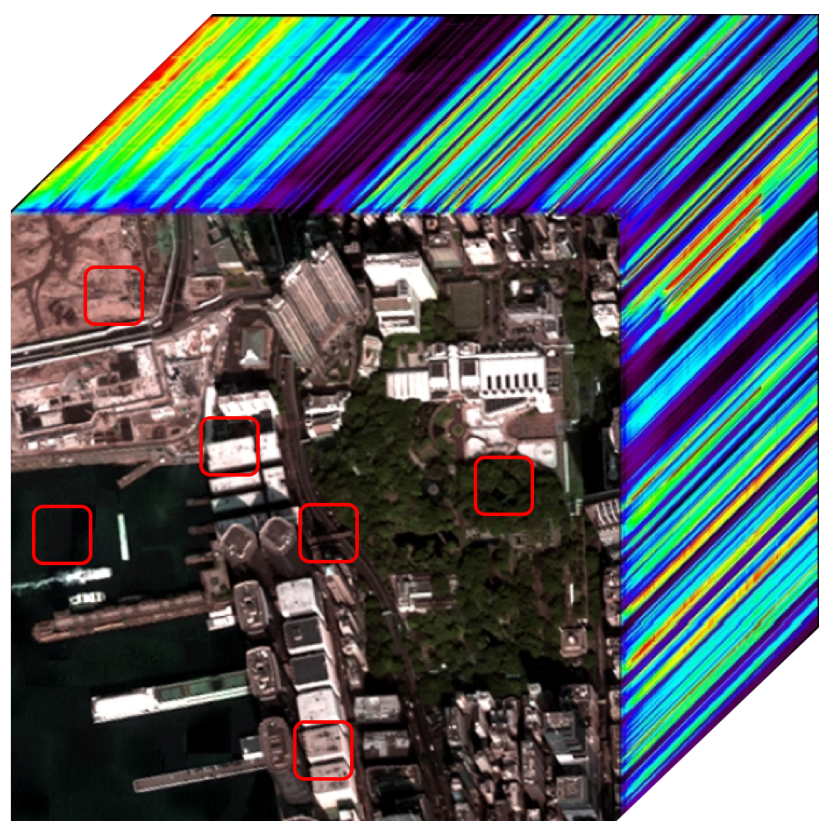

Fig. 6. Hyperspectral cube of a zoomed area from the fused data. 
Out of the main compositions of the research zone, spectra of six types of material are displayed especially for three typical kinds of impervious surfaces. The spectral curves extracted from the fused image are shown in Fig. 7.

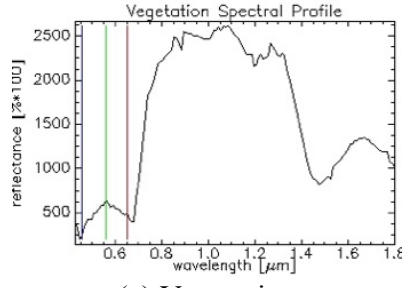

(a) Vegetation

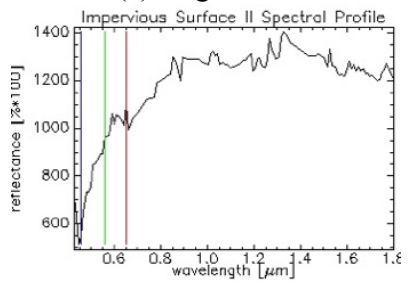

(c) Impervious surface II

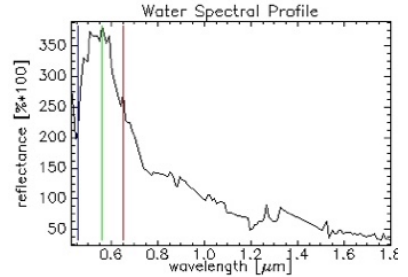

(e) Water

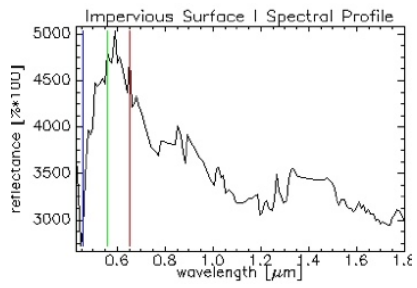

(b) Impervious surface I

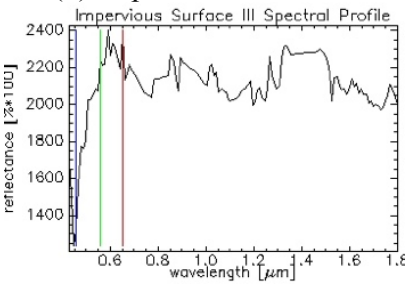

(d) Impervious surface III

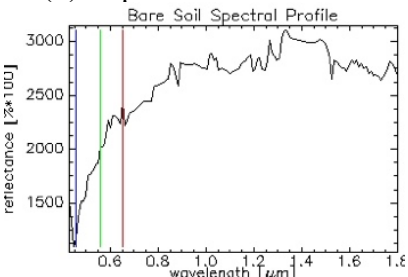

(f) Bare Soil
Fig. 7. Spectral profile of presentative material.

Though the spectra extracted from the fused image could not satisfy the need of quantitative spectral analysis as both the HS and MS have not been strictly radiometric corrected and some of the hyperspectral bands were abandoned due to low signal-tonoise ratio and water absorption. The digital number instead of surface reflectance could still embody some prominent characteristics of these typical materials which could hardly be recognized by on MS image. We have also used one of the benchmark endmember extraction methods, SMACC [5], to extract typical endmembers from this fused image. The sequentially extracted spectrum of first six endmembers are shown in Fig. 8 (a) and their continuums are then removed and shown in Fig. 8 (b).

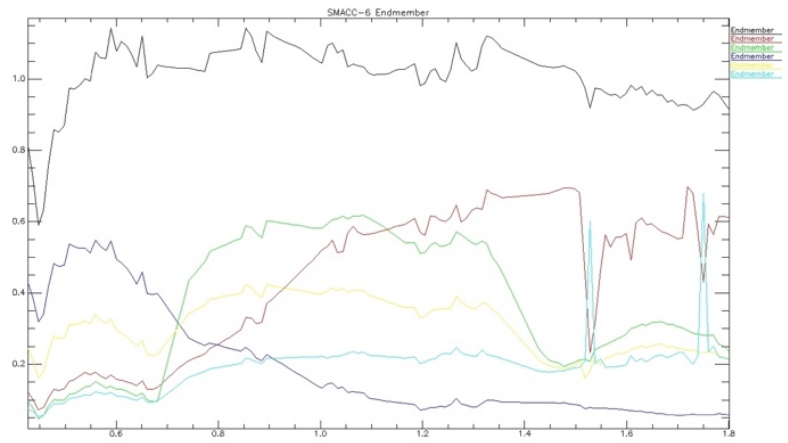

(a) SMACC endmember extraction results from the fused hyperspectral image.

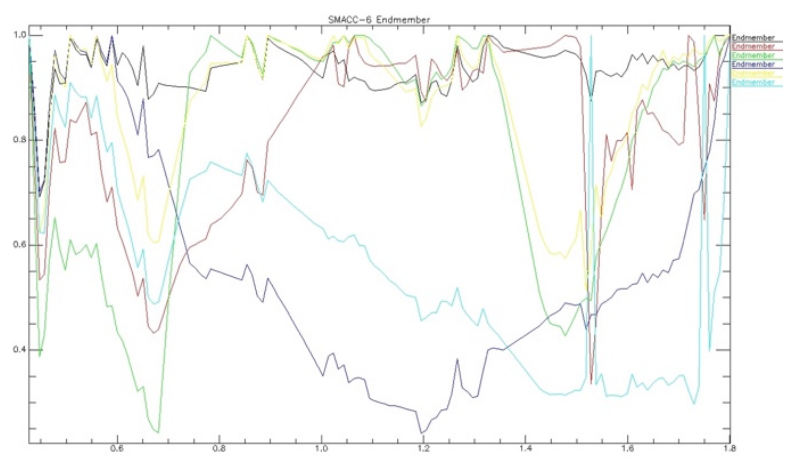

(b) Endmember spectral profile after continuum removal.

Fig. 8. Endmember extraction results.

SMACC also provide with abundances of each endmember. With these carefully chose training samples, using SVM as a classifier, classification results into 7 typical classes of fused image are shown in Fig. 9. Six mentioned classes with typical spectral profile have been well separated even in the case that adjacent roof tops are made of different materials. The shadow areas caused by skyscrapers are unavoidable in high resolution image and they are post classified to one class with unknown characteristics.

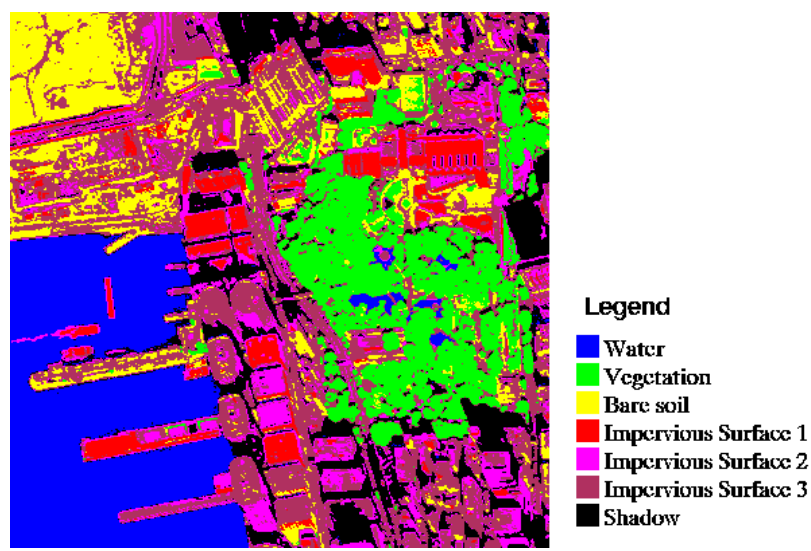

Fig. 9. Classification result with SVM classifier.

For the evaluation of urban ecology status, fused imagery could provide with the relative quantitative estimation result of vegetation health from richer bands. As shown in Fig. 10, the higher level, the healthier of the vegetation status. It is useful for urban ecological monitoring and management.

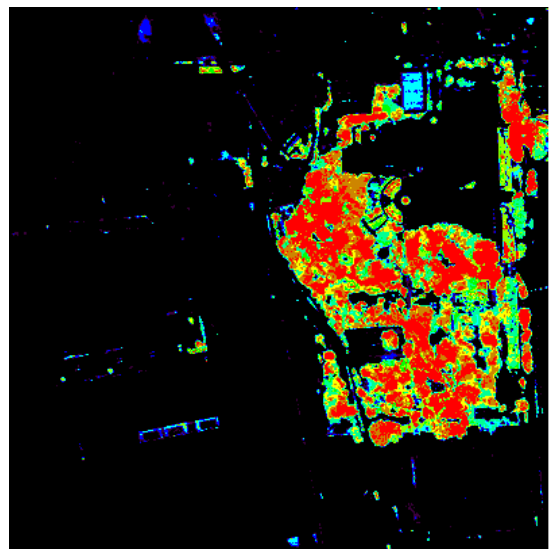

Legend

Non Vegetation

Forest IIealth 1

Forest IIcalth 3

Forest IIealth 4

Forest IIcalth 5

Forest IIcalth 6

Forest IIcalth 7

Forest Ilealth 8

Forest IIealth 9

Fig. 10 Forest health index analysis of fused urban image. 


\section{DISCUSSION}

Hyperspectral imageries have been proved to be sensitive to subtle spectral differences thus capable to exquisitely discriminate these similar materials while limited to the low spatial resolution. Fusion of HSI and MSI provide a promising new way of extracting urban impervious surface and evaluating urban ecological environment at a finer and comprehensive scope The fusion has a great potential in generating richer spectral characteristics for certain kind of land cover that MSI could not provide with, therefore, once properly conducted, it could be applied to various of types of application in the complex urban area.

However, as mentioned in the methodology section, the coregistration between two images with sharp spatial resolution differences is extremely challenging as tie points are difficult to find and match. In addition, low SNR of hyperspectral sensors like SWIR bands of Hyperion will lead to spectra distortion in abundance estimation in CNMF workflow, which will lead to a spectral profile inaccuracies in the fused image.

\section{REFERENCES}

C. Small, "High Spatial Resolution Spectral Mixture Analysis of Urban Reflectance," Remote Sens. Environ., vol. 88, pp. 170$186,2003$.

R. L. Powell, D. A. Roberts, P. E. Dennison, and L. L. Hess, "Sub-pixel Mapping of Urban Land Cover Using Multiple Endmember Spectral Mixture Analysis: Manaus, Brazil," Remote Sens. Environ., vol. 106, pp. 253-267, 2007.

Q. Weng, "Remote Sensing of Impervious Surfaces in the Urban Areas: Requirements, Methods, and Trends," Remote Sens. Environ. , vol. 117, pp. 34-49, 2012.

E. T. Slonecker, D. B. Jennings, and D. Garofalo, "Remote Sensing of Impervious Surfaces: A Review," Remote Sensing Reviews, vol. 20, pp. 227-255, 2001.

Y. Zhang, H. Zhang, and H. Lin, "Improving the Impervious Surface Estimation with Combined Use of Optical and SAR Remote Sensing Images," Remote Sens. Environ., vol. 141, pp. 155-167, 2014.

C. $\mathrm{Wu}$ and A. T. Murray, "Estimating Impervious Surface Distribution by Spectral Mixture Analysis," Remote Sens. Environ., vol. 84, pp. 493-505, 2003.

J. Townshend, C. Justice, W. Li, C. Gurney, and J. McManus, "Global Land Cover Classification by Remote Sensing: Present Capabilities and Future Possibilities," Remote Sens. Environ. , vol. 35, pp. 243-255, 1991.

K. C. Seto, C. E. Woodcock, C. Song, X. Huang, J. Lu, and R. K. Kaufmann, "Monitoring Land-Use Change in the Pearl River Delta Using Landsat TM," Int. J. Remote Sens. , vol. 23, pp. 1985-2004, 2002.

Q. Weng and P. Fu, "Modeling Annual Parameters of Clear-Sky Land Surface Temperature Variations and Evaluating the Impact of Cloud Cover Using Time Series of Landsat TIR Data," Remote Sens. Environ., vol. 140, pp. 267-278, 2014.

T. Wang, H. Zhang, and H. Lin, "High-Level Impervious Surfaces Classification in Urban Environments from
Hyperspectral Imagery," presented at the WHISPERS, Los Angeles, USA, 2016.

C. Small, "Estimation of Urban Vegetation Abundance by Spectral Mixture Analysis," Int. J. Remote Sens., vol. 22, pp. 1305-1334, 2001.

F. Melgani and L. Bruzzone, "Classification of Hyperspectral Remote Sensing Images with Support Vector Machines," IEEE Trans. Geosci. Remote Sens., vol. 42, pp. 1778-1790, 2004.

J. S. Pearlman, P. S. Barry, C. C. Segal, J. Shepanski, D. Beiso, and S. L. Carman, "Hyperion, a space-based imaging spectrometer," IEEE Trans. Geosci. Remote Sens., vol. 41, pp. 1160-1173, 2003.

M. T. Eismann and R. C. Hardie, "Hyperspectral resolution enhancement using high-resolution multispectral imagery with arbitrary response functions," IEEE Trans. Geosci. Remote Sens. , vol. 43, pp. 455-465, 2005.

N. Yokoya, C. Grohnfeldt, and J. Chanussot, "Hyperspectral and Multispectral Data Fusion: A Comparative Review," IEEE Geoscience and Remote Sensing Magazine, vol. 5, pp. 29-56, 2017.

N. Yokoya, T. Yairi, and A. Iwasaki, "Coupled Nonnegative Matrix Factorization Unmixing for Hyperspectral and Multispectral Data Fusion," IEEE Trans. Geosci. Remote Sens. , vol. 50, pp. 528-537, 2012.

D. D. Lee and H. S. Seung, "Learning the Parts of Objects by Non-Negative Matrix Factorization," Nature, vol. 401, pp. 788$791,1999$.

S. Jia and Y. Qian, "Constrained Nonnegative Matrix Factorization for Hyperspectral Unmixing," IEEE Trans. Geosci. Remote Sens., vol. 47, pp. 161-173, 2009.

V. P. Pauca, J. Piper, and R. J. Plemmons, "Nonnegative matrix factorization for spectral data analysis," Linear algebra and its applications, vol. 416, pp. 29-47, 2006.

J. H. Gruninger, A. J. Ratkowski, and M. L. Hoke, "The sequential maximum angle convex cone (SMACC) endmember model," in Defense and Security, 2004, pp. 1-14. 